\title{
Intégration de la radioprotection à la conception de l'usine MELOX*
}

\author{
R. DUCROUX, R. LORENZELLI**
}

\begin{abstract}
RÉSUMÉ L'intégration de la radioprotection à la conception de l'usine MELOX (fabrication de combustible MOX) nous a conduit à définir une méthodologie rigoureuse concernant les études de radioprotection. Nous essaierons de montrer dans cet article comment la radioprotection a été intégrée dans les principes de conception de l'usine par le choix de grandes options (minimiser la durée des opérations normales de fonctionnement, minimiser les durées et les fréquences d'intervention, minimiser les débits d'équivalents de dose) et la fixation des critères de dimensionnement (objectif d'équivalent de dose inférieur à $5 \mathrm{mSv}$ par an). Le poste de broyage, en raison de sa simplicité plus démonstrative, a été choisi à titre d'exemple.
\end{abstract}

ABSTRACT The integration of radiation protection to the MELOX plant design (MOX fuel manufacturing) led us to define a rigorous methodology relating to radiation protection studies.

This paper describes how radiation protection has been integrated in the basic design by choosing major options to minimise intervention lengths and frequencies and equivalent dose rates and setting up design criteria dose equivalent rates lower than $5 \mathrm{mSv} / \mathrm{year}$. The grinding operation, simple but demonstrative for this purpose, has been taken as an illustration.

\section{INTRODUCTION}

L'usine MELOX contribue au recyclage, dans les centrales électronucléaires équipées de réacteurs à eau légère, du plutonium qui se forme dans le cœur des réacteurs au cours de l'irradiation du combustible à $\mathrm{UO}_{2}$ faiblement enrichi, et permet de valoriser ainsi l'utilisation de l'uranium. Elle intervient, à ce titre, dans le cycle du combustible comme complémentaire d'installations existantes, telles que celles de retraitement des combustibles irradiés dont elle constitue un prolongement. Elle est destinée à la fabrication de combustible à oxyde mixte d'uranium et de plutonium (MOX) et produira industriellement $120 \mathrm{t}$ de combustible sous forme d'assemblages.

Cette usine est implantée en limite de l'établissement COGEMA de Marcoule. Elle couvre une superficie d'environ 5 ha et est constituée de deux bâtiments nucléaires (fabrication-traitement des rebuts et déchets)

\footnotetext{
* Communication présentée lors des journées SFRP, "Les nouveaux enjeux de la radioprotection des travailleurs", Lyon, 11-12 juin 1991.

** MELOX, 116, avenue A.-Briand, 92220 Bayeux
} 
et de bâtiments annexes. La mise en service de cette usine est prévue en fin d'année 1994.

Les objectifs de dose que l'on s'est fixés pour la conception de l'usine MELOX montrent une volonté de faire en sorte que les équivalents de dose reçus par le personnel se situent bien en deçà des valeurs légales [1]. En effet, la démarche retenue a été de fixer comme objectif d'avoir, dans les conditions normales d'exploitation, un nombre d'agents aussi faible que possible susceptibles de recevoir un équivalent de dose dépassant $5 \mathrm{mSv} / \mathrm{an}$. Cet objectif de limitation des doses des agents s'est traduit dans les principes de conception des installations, mais a entraîné également une méthodologie rigoureuse concernant les études de radioprotection [3]. Nous essayerons de montrer, à cet effet, comment la radioprotection a été intégrée dans les principes de conception de l'usine par le choix de grandes options et la fixation des critères de dimensionnement.

\section{PRINCIPES DE CONCEPTION DE L'USINE}

Le principe général retenu est d'avoir une ligne de produits avec des stockages intermédiaires donnant une grande souplesse d'exploitation (fig. 1). Le travail des matières est effectué par charge discrète dans des conteneurs, différents suivant l'endroit où est situé le produit : trémie, broyeur, jarre, homogénéiseur... pour les poudres ; boîtes, plateaux... pour les pastilles ; tubes pour les crayons.

Les postes de travail sont situés en boîtes à gants (BAG) dont le volume varie de quelques mètres cubes à $25 / 30 \mathrm{~m}^{3}$ suivant la complexité du poste. Ces BAG sont reliées entre elles par des tunnels de manutention où des convoyeurs font passer le produit d'un poste à l'autre. Les BAG sont également connectées avec les unités de stockage intermédiaire, ce qui évite le blocage d'un poste amont ou aval en cas de panne.

La conduite normale des postes de travail est reportée en salle de conduite centralisée. Les opérateurs, par l'intermédiaire d'un système informatisé, gèrent le fonctionnement du poste. Certains équipements nécessitent une présence temporaire d'opérateurs auprès des postes pour effectuer des opérations normales de fonctionnement (ONF) mais également des interventions sur incident de fonctionnement (IIF) nécessaires pour maintenir le bon fonctionnement du poste. Le bon déroulement des opérations du poste est également contrôlé aux points sensibles par des équipements de surveillance de type caméra. 


\begin{tabular}{|c|c|c|c|}
\hline ATELIER & FOMCTION8 & ARCHITECTURA & $\begin{array}{l}\text { QUANTITES } \\
\text { ANNUELLES }\end{array}$ \\
\hline POUDRE8 & $\begin{array}{l}\text { STOCKAGE PUCQ et UO2 } \\
\text { DEBOITAGE-DOSAGE } \\
\text { BROYAGE } \\
\text { DOSAGE MELANGE } \\
\text { BECONDAPE } \\
\text { STOCKKAGE POUDRE } \\
\text { HOMOGEMEISATION } \\
\text { GRANULATION } \\
\text { PASTILLAGE }\end{array}$ & & $120 t$ \\
\hline PA8TLLEB & 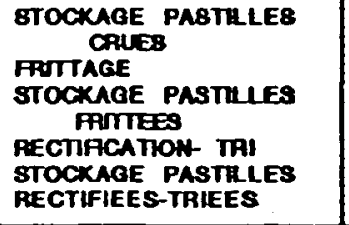 & & $\begin{array}{c}2,5 \\
\text { Millions } \\
\text { pastilles }\end{array}$ \\
\hline & $\begin{array}{l}\text { CAIMAGE } \\
\text { DECONTAMIMATION }\end{array}$ & & \\
\hline CRAYONS & 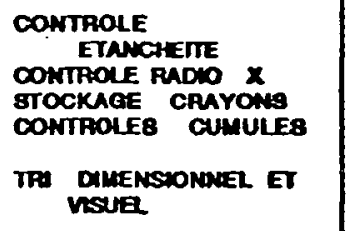 & & $\begin{array}{l}66000 \\
\text { crayons }\end{array}$ \\
\hline ASSEMBLAGES & $\begin{array}{l}\text { MONTAGE ASSEMELACE } \\
\text { COATROLE } \\
\text { ASSEMBLLAE } \\
\text { BTOCXAGE ABSEMBLACE }\end{array}$ & & 250 \\
\hline
\end{tabular}

Fig. 1. - Organigramme de la production.

\section{OPTIONS DE LA RADIOPROTECTION}

Les grandes options de la radioprotection ont été de minimiser :

- les durées des opérations normales de fonctionnement nécessitant la présence d'opérateurs,

- les durées et les fréquences d'intervention,

- les débits d'équivalents de dose (DED).

\section{Minimiser les durées des ONF}

La recherche de la diminution des durées des ONF s'est effectuée dans deux directions ; l'une basée sur l'aménagement des conditions de travail, l'autre basée sur le retour d'expérience des usines de fabrication des combustibles. 


\section{L'aménagement des conditions de travail}

Les conditions de travail ont été étudiées de façon à simplifier les tâches de l'opérateur à chaque opération normale de fonctionnement. Tout d'abord, l'accès au lieu d'opération est facilité par des "chemins de moindre dose". Au poste de travail, des passerelles protégées permettent de passer d'un niveau à l'autre en fonction de la hauteur des BAG. Le lieu du poste où se déroule l'opération est soigneusement étudié pour protéger l'opérateur contre l'exposition due aux postes voisins et au rayonnement diffusé.

Par ailleurs, afin de réduire les durées des ONF, des dispositifs ont été créés pour alléger le nombre d'opérations et les simplifier le plus possible dans leur exécution. Sans rentrer dans les détails, on citera à titre d'exemples :

- l'introduction d'additifs à la poudre d'oxyde mixte dans le mélangeur : utilisation de sacs prédosés en dehors des zones à risque d'exposition et introduction dans le poste au moyen de dispositif mécanisé étanche ;

- l'aménagement des postes de contrôle de la qualité des pastilles ;

- l'aménagement des contrôles visuels de la qualité des soudures des crayons, de l'assemblage, au moyen de caméras.

\section{Le retour d'expérience}

Le procédé retenu est utilisé depuis quelques années dans l'usine de Belgonucléaire à Dessel. De même, le complexe de fabrication des combustibles à Cadarache (unité COGEMA) a une longue expérience dans la fabrication des combustibles à base de plutonium.

Le retour d'expérience a été pris en compte au niveau :

- de la conception des équipements (broyage, pressage, mélange...),

- des scénarios d'exploitation,

- de l'amélioration des niveaux d'exposition (en utilisant les mesures dosimétriques).

Cela s'est traduit par la mise en place de protections au plus près des équipements, par l'utilisation de systèmes de dépoussiérage, par l'automatisation partielle, voire totale, de certains équipements tels que le tri d'aspect des pastilles ou l'inspection des crayons qui ont fait l'objet de prototypes essayés en production industrielle.

\section{Minimiser les durées et les fréquences des IIF}

La conception prend en compte les contraintes d'accessibilité et de visibilité. Tous les éléments constituant l'équipement du poste sont, dans la mesure du possible, modulaires et facilement interchangeables dans des conditions ergonomiques. La standardisation des équipements parti- 
cipe également, dans une large mesure, à l'interchangeabilité des pièces ou des éléments d'équipements. La conception de ces équipements doit être simple et fiable. Ce paramètre est d'autant plus difficile à mettre en œuvre que l'automatisation est poussée. La fiabilité des technologies adoptées doit être éprouvée.

\section{Minimiser les DED}

Pour chaque poste (BAG et équipement associé) de l'usine, est conduite une étude de dimensionnement des protections biologiques qui permet de prévoir :

- des protections internes implantées au plus près des sources autour des équipements et, en règle générale, la quantité maximale de protection compatible avec les autres impératifs de conception tels que la thermique, la mécanique, le poids, les dimensions...

- des protections externes accrochées sur les boîtes à gants ; leur localisation et leurs dimensions sont déterminées pour atteindre l'objectif de dose en fonction du temps de présence des opérateurs.

Cette étude qui sert au prédimensionnement des postes de travail de l'usine est basée sur des critères qui sont soit imposés, soit variables.

\section{DONNÉES D'ÉTUDE DE DIMENSIONNEMENT}

\section{Critères imposés}

Les études de radioprotection ont pour but de limiter, à la conception, le nombre d'agents qui dépasserait $5 \mathrm{mSv} / \mathrm{an}$. Cette limitation, dans des conditions normales de fonctionnement, a été traduite par la relation :

$$
\frac{\sum_{i} f_{i} t_{i} D_{i}}{\sum_{i} f_{i} t_{i}} \leq \frac{5}{T} \text { en } \mathrm{mSv} / \mathrm{h}
$$

avec : $f_{i}$ fréquence d'intervention/an

$t_{i}$ temps d'intervention (en $\mathrm{h}$ )

$D_{i}$ débit d'équivalent de dose (en $\mathrm{mSv} / \mathrm{h}$ )

$T$ durée annuelle de travail (en $h$ ).

\section{Critères variables}

Par contre, les données suivantes retenues pour les études sont des valeurs qui peuvent être modulables selon les cas critiques rencontrés :

- une donnée concerne le débit d'équivalent de dose ambiant fixé à $25 \mu \mathrm{Sv} / \mathrm{h}$ à $1 \mathrm{~m}$ autour des postes, cette donnée n'est, en général, dimensionnante que pour les postes ayant peu d'ONF ; 
- les interventions (IIF) doivent être effectuées avec un DED n'excédant pas $50 \mu \mathrm{Sv} / \mathrm{h}$, la valeur instantanée ne doit pas dépasser $1 \mathrm{mSv} / \mathrm{h}$; seules les interventions fréquentes et occasionnelles sont étudiées; les interventions sont considérées comme exceptionnelles lorsque leur fréquence annuelle est $<0,5$; les interventions aux limites sont traitées au cas par cas ;

- la contribution des postes voisins ainsi que la capacité annuelle transitant dans le poste sont prises en compte au niveau de la relation (1) :

à un poste $k$ avec une contribution d'un poste $j$

$$
\frac{\sum_{i} t_{i} f_{i}\left(D^{\mathrm{m}}{ }_{k i}+\sum_{j} D^{\mathrm{m}} j i \frac{C_{\mathrm{m} j}}{C_{\mathrm{n} j}}\right)}{\sum_{i} f_{j} t_{i}} \leq \frac{5}{T} \text { en } \mathrm{mSv} / \mathrm{h}^{-1}
$$

$C_{\mathrm{n}}$ : capacité nominale journalière $\mathrm{x}$ nombre de jours de production

$C_{\mathrm{m}}$ : capacité moyenne annuelle

$D_{k i}^{\mathrm{m}}$ : DED (moyenné sur l'opération $l$ ) provenant des sources du poste $k$ reçu par l'opérateur $i$ sur le poste $k$ :

$$
D_{k i}^{\mathrm{m}}=\frac{1}{t_{i}} D_{i} \mathrm{~d} t
$$

$D^{\mathrm{m}}{ }_{j i}$ : DED (moyenné sur le cycle de fonctionnement) provenant $\mathrm{du}$ poste $j$ voisin du poste $k$ où est effectué l'opération $i$.

$$
D_{j i}^{\mathrm{m}_{j i}}=\frac{1}{t_{j}} D_{j} \mathrm{~d} t
$$

\section{Qualité des matières traitées}

Les calculs de radioprotection ont été effectués avec des poudres de $\mathrm{PuO}_{2}$ issues du retraitement de combustible $\mathrm{UO}_{2}$ irradié (33 $000 \mathrm{MWJ} / \mathrm{t}$ ) vieillies 6 ans, ce qui correspond à une teneur en américium 241 de 30000 ppm, valeur limite acceptée par MELOX.

$\mathrm{Ce} \mathrm{PuO}_{2}$ est enveloppe vis-à-vis d'un $\mathrm{PuO}_{2}$ provenant de combustibles irradiês à $45000 \mathrm{MWJ}^{-1}$ vieilli 3,5 ans. Les teneurs moyennes de plutonium total prises en compte dans les calculs sont de $30 \%$ (Pu/U + $\mathrm{Pu}$ ) au niveau des poudres de mélange mère (tête de procédé) et de $10 \%$ pour les autres postes de fabrication jusqu'à l'expédition des assemblages.

Les rayonnements et particules considérés dans les calculs sont les gamma, les neutrons et, pour quelques cas (source sans aucune protection), les $X$ de fluorescence provenant de l'uranium et du plutonium. 


\section{Méthodologie des études}

Elle repose sur une étude détaillée du poste. L'ensemble des tâches d'exploitation est décomposé en tâches élémentaires correspondant à l'exposition d'un opérateur en un point, pour une situation et un temps donnés. II "suffit" d'estimer le DED en ce point comme somme des DED élémentaires qui sont les contributions de chaque source en débit de dose à travers des protections biologiques supposées définies. Des itérations sont effectuées afin d'obtenir le respect des critères de dimensionnement.

\section{Sources, calculs et codes}

Compte tenu de la répétition des sources le long de la ligne de fabrication, un fichier de sources standards a été établi à l'aide des codes TRIPOLI, MERCURE 4 et INTERPOL [2]. Le nombre de points de calculs nécessaires à l'évaluation des DED nous a conduit à élaborer un code de calcul nommé ACORA (assistance à la conception de la radioprotection) développé par le Centre d'étude pour l'évaluation de la protection nucléaire (CEPN).

Ce code prend en compte :

- les variations des masses de matière nucléaire dans le poste (CALMAS VARMAS),

- les hétérogénéités dans les protections et les équipements (CALVIDE),

- les calculs à travers les ronds de gants des BAG,

- la contamination superficielle dans les BAG,

- les contributions des rayonnements diffusés par les sol et plafond,

- les rétentions de matière dans les équipements,

- la mobilité des sources dans le poste,

- les traversées multiples,

- la contribution des stockages et des postes voisins.

L'organisation des calculs est donnée dans la figure 2. Elle montre la grande souplesse du code ACORA qui, hormis le fichier de sources standards, permet de modifier toutes les données d'entrée et, par itération, de définir le bon dimensionnement des protections biologiques, mais également de fournir une analyse variationnelle donnant un grand choix de solutions. 


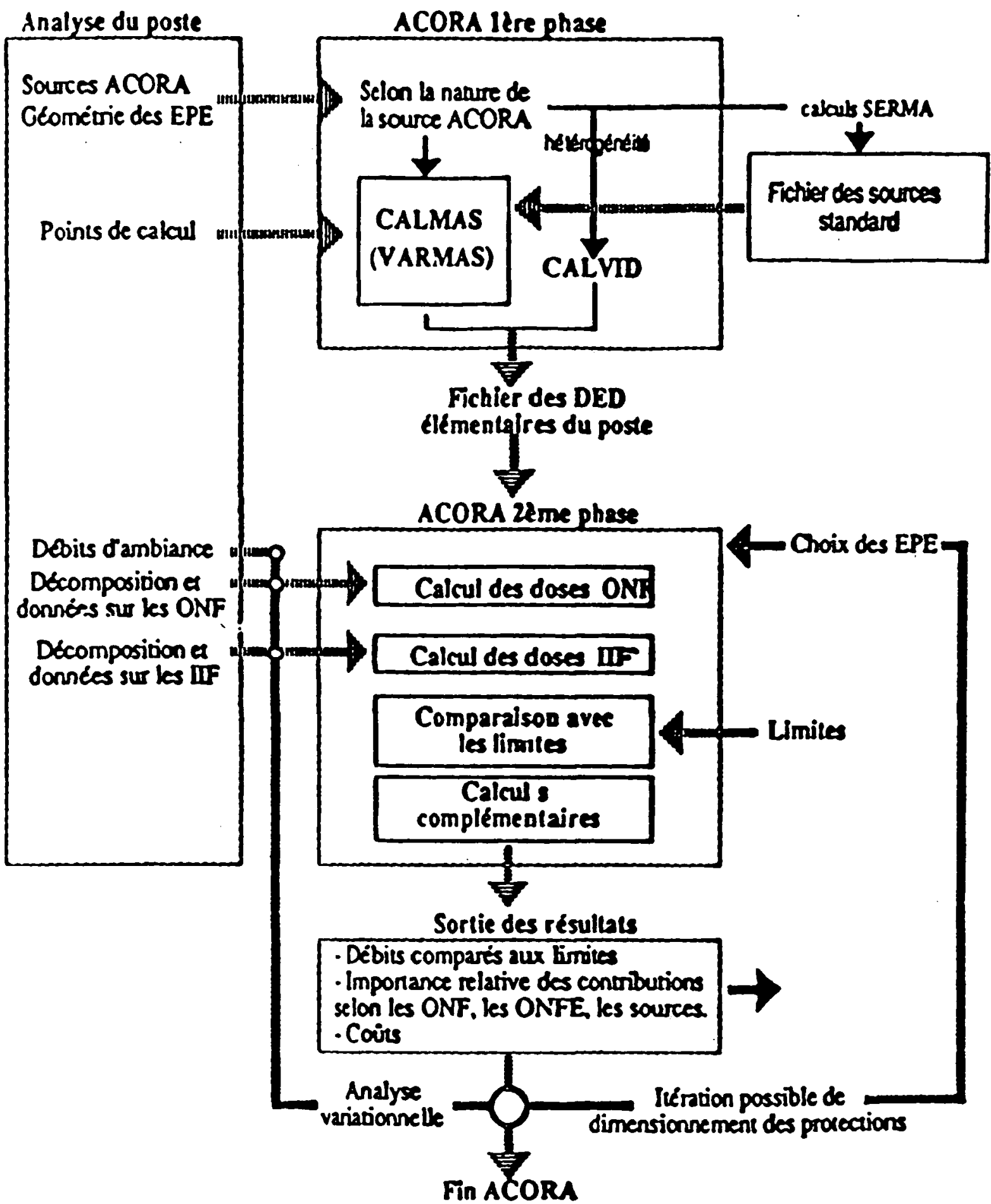

ONF : Opération normale de fonctionnement ; IIF : Intervention sur incident de fonctionnement ; EPE : Ecran de protection externe

Fig. 2. - Logigramme du programme ACORA. 


\section{Exemple d'application}

Nous avons choisi comme exemple le poste de broyage en raison de sa simplicité plus démonstrative. La fonction principale du poste est d'assurer le broyage du mélange mère à $30 \%$ de $\mathrm{PuO}_{2}$ dans le but d'obtenir une micro-homogénéité de répartition du $\mathrm{PuO}_{2}$ dans $\mathrm{I}^{\prime} \mathrm{UO}_{2}$.

Les fonctions principales sont :

- la manutention des jarres, pesée, identification...

- l'accostage et le désaccostage jarre-broyeur,

- le changement des hottes de protections biologiques des jarres.

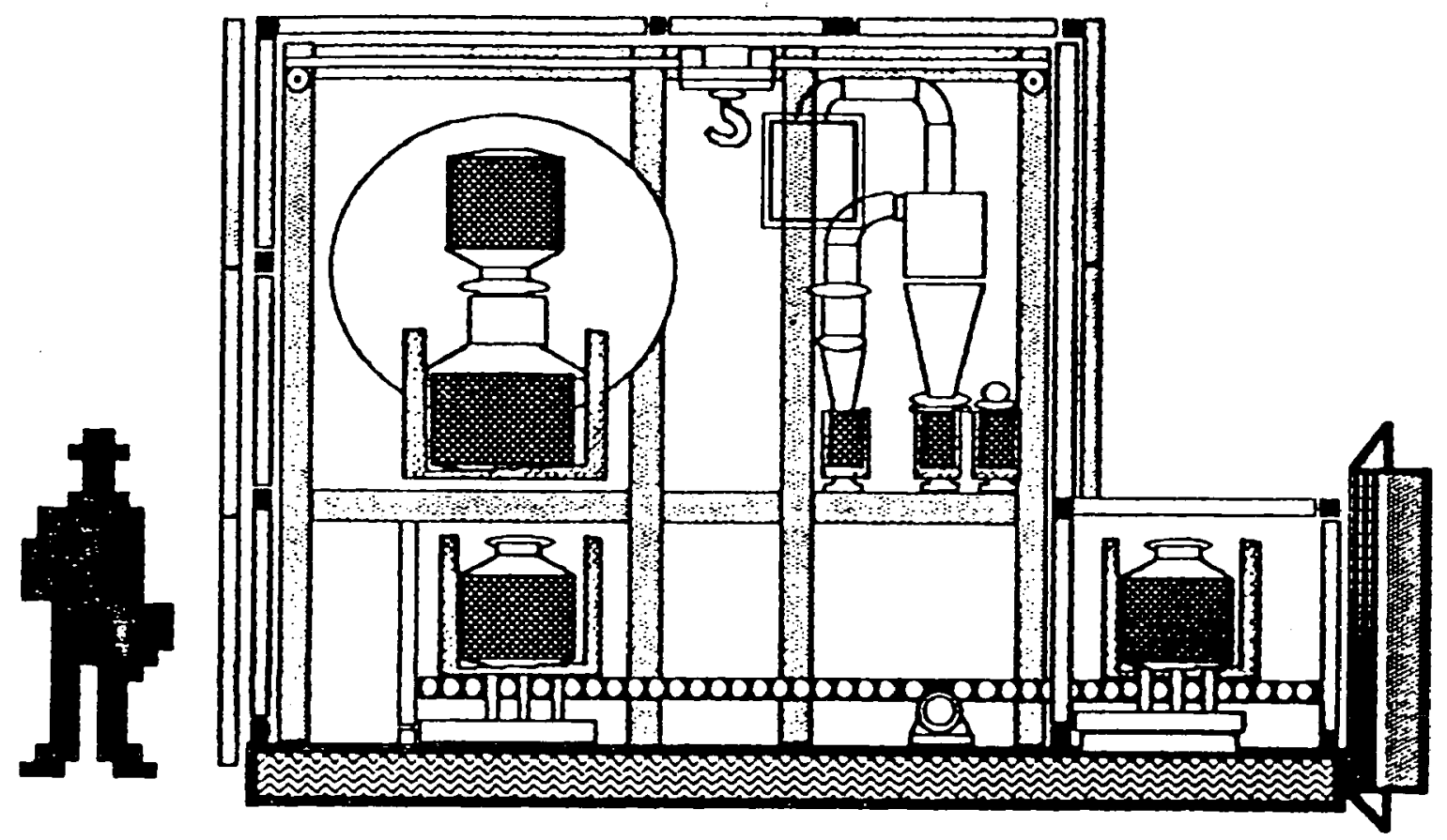

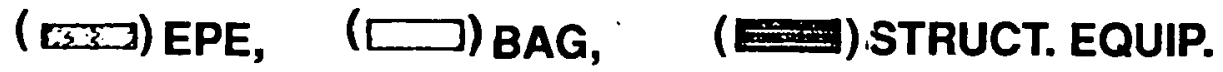

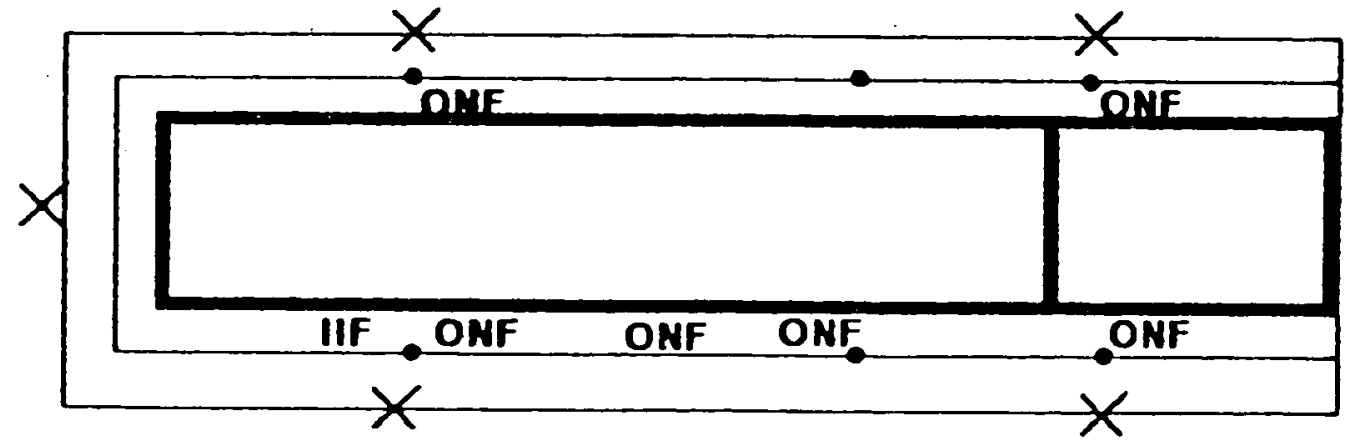

(X) Ambiance (1 =), (•) Surveillance locale $(50 \mathrm{~cm})$

Fig. 3. - Le broyage. 
Le poste fonctionne entièrement en automatique. Un cycle de fonctionnement est composé d'environ 24 opérations. La figure 3 donne le positionnement des sources et définit la localisation des protections biologiques internes.

Les ONF sont identifiées et estimées en durées, fréquences, position opérateur et sources en présence. Elles sont constituées par le changement des pots de poussières, le changement des filtres de dépoussiérage, la surveillance en local, les arrêts intercampagnes, la maintenance $1^{\text {er }}$ niveau, la circulation entre zones et le changement des filtres d'enceinte.

Les IIF (panne de balance, du déboucheur reboucheur, convoyeur à rouleaux, broyeur...), avec un scénario d'intervention qui prend en compte une configuration moyenne des charges dans le poste, sont également identifiées.

Après l'identification détaillée de la géométrie et du fonctionnement du poste, les données pour le calcul sont saisies dans ACORA. Par ailleurs, les sources ont été calculées (matière associée au conteneur avec sa protection biologique). Dans ce cas, une des difficultés réside dans le choix des positions du broyeur à considérer dans les calculs. En effet, celui-ci est animé d'un mouvement complexe qui engendre des DED dans diverses directions. Un calcul de rayonnement diffusé a été effectué pour repérer les directions pénalisantes. Les résultats de calcul sont donnés dans le tableau I.

TABLEAU I

Résultats pour les conditions initiales de calcul tout élément de protection extérieure (EPE) en kiowaglass $(2 \mathrm{~cm})$

\begin{tabular}{|c|c|c|}
\hline W & $\begin{array}{l}\text { Limites a respecter } \\
\text { en } \mathrm{SSv} / \mathrm{h} \text { (mrem/h) }\end{array}$ & $\begin{array}{l}\text { Résultats } \\
\text { en } \mu S v / h\end{array}$ \\
\hline ONF & 4,45 en moyenne $(0,45)$ & Limite respectée 4,45 en moyenne \\
\hline Limite à $1 \mathrm{~m}$ & 25 en tout point $(2,5)$ & $\begin{array}{l}\text { Limite respectée en tout point dans } \\
\text { la fourchette } 12 \text { à } 20\end{array}$ \\
\hline IIF & $\begin{array}{l}\text { a) } 1000 \text { (débit maximal) (100) } \\
\text { b) } 50 \text { (débit moyen) (5) }\end{array}$ & $\begin{array}{l}\text { Limite respectée pour une IIF } \\
\text { Limite respectée pour une IIF }\end{array}$ \\
\hline
\end{tabular}

Ces résultats montrent qu'avec une épaisseur de protection extérieure de $2 \mathrm{~cm}$ en kiowaglass (métacrylate de métyl dopé au plomb) tous les critères sont respectés. 


\section{CONCLUSIONS}

L'application des grandes options de radioprotection dès la conception d'un projet de réalisation d'une installation nucléaire complexe doit permettre de s'approcher, en cours d'exploitation, des objectifs de dose annuelle visés en cours d'étude. Cependant, ces objectifs ne peuvent être atteints que par le passage obligé d'un lourd investissement aussi bien au niveau des études que de la réalisation.

\section{RÉFÉRENCES}

[1] R. COATES. - Experience on the application of the ALARA principle. J. Radiol. Prot., 1990,10 (1) 39-42

[2] C. DUPONT. - MERCURE 4 : un programme de Monte Carlo à 3 dimensions pour l'intégration de noyaux ponctuels, atténuation en ligne droite. (Rapport DEMT 85341). Saclay : Commissariat à l'énergie atomique, DEMT, 1985.

[3] P. PAGES, J.P. DEGRANGE, J.C. NIMAL, Y.K. LEE, R. LORENZELLI, R. DUCROUX. - ALARA at the design stage of nuclear installations. In : Conference on occupational radiation protection, Guernesey, 29 April-3 May 1991, Londres : British nuclear energy, 1991, 83-86. 